\title{
TWO THEOREMS ON TRUTH TABLE DEGREES
}

\author{
R. G. DOWNEY
}

(Communicated by Thomas J. Jech)

\begin{abstract}
In this article we solve two questions of Odifreddi on the r.e. tt-degrees. First we construct an r.e. tt-degree with anticupping property. In fact, we construct r.e. tt-degrees $\mathbf{a}, \mathbf{b}$ with $\mathbf{0}<\mathbf{a}<\mathbf{b}$ and such that for all (not necessarily r.e.) tt-degrees $\mathbf{c}$ if $\mathbf{a} \cup \mathbf{c} \geq \mathbf{b}$ then $\mathbf{a} \leq \mathbf{c}$. This result also has ramifications in, for example, the r.e. wtt-degrees. Finally we solve another question of Odifreddi by constructing an r.e. tt-degree with no greatest r.e. $m$-degree.
\end{abstract}

1. Introduction. The goal of this paper is to answer two questions of Odifreddi concerning r.e. tt-degrees. For background we refer to Odifreddi $[\mathbf{9}, 10]$ or Rogers [12]. The relevant questions are:

(1.1) [9, Problem 11] Does every r.e. tt-degree have greatest r.e. $m$-degree.

(1.2) Does $\mathbf{0}_{\mathrm{tt}}^{\prime}$ have the anticupping property? Namely, is there an r.e. tt-degree $\mathbf{a} \neq \mathbf{0}$ such that for all r.e. tt-degrees $\mathbf{b}$, if $\mathbf{b} \cup \mathbf{a}=\mathbf{0}_{\mathrm{tt}}^{\prime}$ then $\mathbf{b}=0_{\mathrm{tt}}^{\prime}$ ?

We solve (1.1) negatively. The method extends to construct on r.e. tt-degree a containing no $n$-r.e. $m$-degree exceeding all r.e. $m$-degrees in a.

We solve (1.2) affirmatively. This question was particularly interesting in view of the fact that the analogous questions had been solved for all other (major) reducibilities. The method we use is very different from those used for other reducibilities. Although it is not difficult this method is quite powerful. In fact we are able to show that $\mathbf{0}_{\mathrm{tt}}^{\prime}$ has the global anticupping property: let $\mathscr{D}_{\mathrm{tt}}$ denotes the collection of all tt-degrees. We say an r.e. tt-degree $\mathbf{d} \neq \mathbf{0}$ has the global anticupping property if there exists an r.e. $\mathrm{tt}$-degree $\mathbf{a}$ with $\mathbf{0}<\mathbf{a} \leq \mathbf{d}$ such that

$$
\forall \mathbf{b} \in \mathscr{D}_{\mathrm{tt}}(\mathbf{b} \cup \mathbf{a} \geq \mathbf{d} \rightarrow \mathbf{b} \geq \mathbf{a}) \text {. }
$$

The technique used to establish that $\mathbf{0}_{\mathrm{tt}}^{\prime}=\mathbf{d}$ satisfies (1.3) is also applicable to various other situations and reducibilities. To demonstrate this, a minor variation of the construction establishes a result from [1] : if $\mathbf{d}$ is any nonzero r.e. wtt-degree, there exists an r.e. wtt-degree $\mathbf{a}$ with $\mathbf{0}<\mathbf{a}<\mathbf{d}$ such that for all wtt-degrees $\mathbf{b}$, if $\mathbf{a} \cup \mathbf{b} \geq \mathbf{d}$ then $\mathbf{a} \leq \mathbf{b}$. In particular, all nonzero r.e. wtt-degrees have the global anticupping property. This last result does not hold for the r.e. tt-degrees since, for example, there are minimal r.e. tt-degrees (Kobzev [7]). The reader should note that the analogue of (1.3) does not hold for $\mathbf{d}=\mathbf{0}_{\mathrm{T}}^{\prime}$ in the $\mathrm{T}$-degrees since the upper semilattice of $T$-degrees $\geq \mathbf{0}_{\mathrm{T}}^{\prime}$ is complemented (Posner [11]).

Received by the editors July 15, 1986.

1980 Mathematics Subject (Ylassification (1985 Revision). Primary 03D30, 03D25.

Key words and phrases. Global anticupping property, truth table degrees, $m$-degrees.

This research carried out whilst the author was visiting the University of Illinois at UrbanaChampaign, and was partially supported by NSF grant DMG 86-01242. 
It seems convenient to adopt a variation of the notation of Fejer and Shore [4]. Thus if $\{e\}(x) \downarrow$ then $[e]$ is the truth table with index $\{e\}(x)$. For a set $A$ we define

$$
[e](A ; x)= \begin{cases}1 & \text { if }\{e\}(x) \downarrow \text { and } A \vDash[e](x), \\ 0 & \text { if }\{e\}(x) \downarrow \text { and } \rightarrow(A \vDash[e](x)), \\ \uparrow & \text { if }\{e\}(x) \uparrow .\end{cases}
$$

Similarly, we define $[e]_{s}\left(A_{s} ; x\right)$ according to whether or not $\{e\}_{x}(x) \downarrow$. When the context is clear, we shall write $[e]\left(A_{s} ; x\right)$ for $[e]_{s}\left(A_{s} ; x\right)$ to simplify notation. We let $u(-)$ denote the use of $(-)$. We let $A[x]=\{z \in A: z \leq x\}$, and use $\langle$,$\rangle to denote$ a standard pairing function, we assume $\langle$,$\rangle is monotone in both variables. We$ assume that all computations, etc. are bounded by $s$ at stage $s$. All other notation and terminology is standard and we refer the reader to $[\mathbf{9}, \mathbf{1 0}$, or 12].

The author wishes to thank Carl Jockusch, Barry Cooper and Christine Haught for helpful conversations regarding this material.

\section{Anticupping.}

(2.1) THEOREM. There exists an r.e. tt-degree $\mathbf{d}$ with the global anticupping property.

REMARK. As a corollary we see that $\mathbf{0}_{\mathrm{tt}}^{\prime}$ has the global anticupping property since $\mathbf{0}_{\mathrm{tt}}^{\prime} \geq \mathbf{d}$. However the reader should note that the construction below is more flexible and we can, for instance, make $\mathbf{d}$ low. In the notation of our construction, this involves the use of many "entourages of followers". We do not pursue such variations as they do not seem central to the issues of this paper.

ProOF. We shall construct r.e. sets $A=\bigcup_{s} A_{s}$ and $D=\bigcup_{s} D_{s}$ to satisfy the requirements below

$P_{e}: \bar{A} \neq W_{e}$

$N_{e}$ : If $B$ is any set then $[e](A \oplus B)=D$ implies $A \leq_{\mathrm{tt}} B$.

Additionally, we arrange that $A \leq_{\mathrm{tt}} D$ (in fact the construction ensures $A \leq_{m}$ $D$ ). We shall use $\sigma, \tau, \gamma$ etc. to denote strings (i.e. $\sigma, \tau \in 2^{<\omega}$ ) and $\operatorname{lh}(\sigma)$ will denote the length of $\sigma$. Let

$$
l(e, s)=\max \left\{x: \exists \sigma \forall y<x\left([e]\left(A_{s} \oplus \sigma ; y\right)=D_{s}(y)\right)\right\} .
$$

Define

$$
u(e, y, s)=\left\{\begin{array}{l}
u\left([e]\left(A_{s} \oplus \sigma ; y\right)\right) \quad \text { for any } \sigma \text { with }[e]\left(A_{s} \oplus \sigma ; y\right) \downarrow \text { and } \operatorname{lh}(\sigma) \leq s \\
\uparrow \quad \text { if } \forall \sigma\left(\operatorname{lh}(\sigma) \leq s \rightarrow[e]\left(A_{s} \oplus \sigma ; y\right) \uparrow\right) .
\end{array}\right.
$$

Note that the use of tt-reductions means that it is irrelevant which $\sigma$ we use in the first case above. Now let

$$
\begin{aligned}
m l(e, s) & =\max \{l(e, t): t<s\}, \\
m u(e, s) & =\max \{u(e, y, s): y<l(e, s)\} .
\end{aligned}
$$

We shall satisfy the $P_{e}$ by followers. Each follower of $P_{e}$ is targeted for $A$ and is attached to a unique prefollower targeted for $D$. $E+3$ prefollowers are appointed to $P_{e}$ at the beginning of the construction and are used to satisfy the $N_{j}$ for $j<e$. The number $e+3$ comes from the quantity of $N_{j}$ with which $P_{e}$ must cooperate. (More on this later.) 
We shall first briefly describe our basic strategy for satisfying $N_{e}$. It is important to note that $B$ is unknown during the construction and we must play for all possible $B$. Our fundamental idea-for a single $N_{e}$-is to use the prefollower $y(x)$ of a follower $x$ to force a $B$-predictable change in $D$ via $[e]$. The implementation is roughly as follows.

We have, at each stage, a least unused prefollower $y \in E(j)$ (to be defined later) targeted for $D$. We shall wait until a stage $s$ such that $l(e, s)>y$, declare $P_{j}$ as $e$-confirmed and reset a new follower $x$ of $P_{j}$ targeted for $A$ and attached to $y$. We appoint $x>s$ and so obtain the situation described in the diagram below.

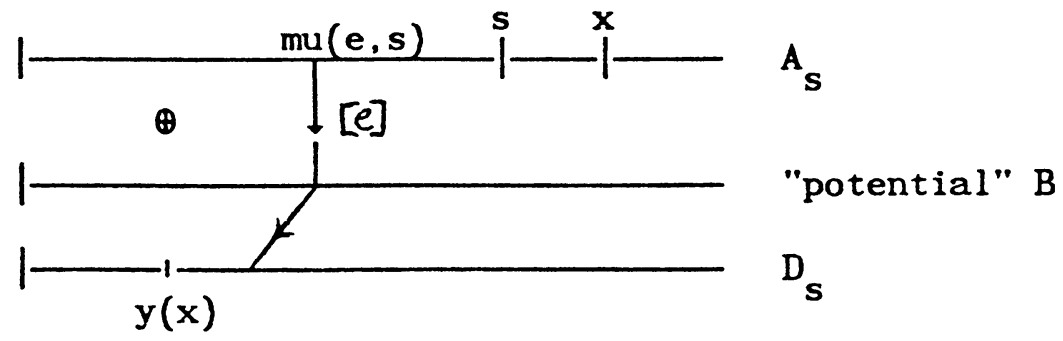

We now promise that for all stages $t \geq s$

$$
x \in A_{t+1}-A_{t} \quad \text { iff } y(x) \in D_{t+1}-D_{t} .
$$

Now suppose that we have built our reduction procedure so that $B$ can tt-compute $A[x-1]$, which we write as " $A[x-1] \leq_{\mathrm{tt}} B$ ". It follows by $(2.2)$ and the fact that $x>m u(e, s)$, that if $[e](A \oplus B)=D$ then

$$
x \in A \quad \text { iff }[e](A[x-1] \oplus B ; y(x))=1 .
$$

But (2.3) and the assumption that $A[x-1] \leq_{t t} B$ mean that $A[x] \leq_{t t} B$. In this way we show that $A \leq_{t t} B$.

The reader should note that it is necessary to use more than one prefollower for a single $P_{e}$ for the following reason. Suppose in the situation of the diagram we are concerned with two $N_{e}$ say $N_{e_{1}}$ and $N_{e_{2}}$. Now when we see $l\left(e_{1}, s\right)<y(x)$ we attached $x$ to $y(x)$. Now this gives us a permanent commitment to enumerate $x$ into $A$ iff $y(x) \in D$. The trouble is that perhaps at some $\hat{s}>s$ for $e_{2}$ it may be that $u\left(\left[e_{2}\right]\left(A_{\hat{s}} \oplus \sigma ; y(x)\right)\right)>x$. This means that $x$ is no longer a good follower for $P_{j}$ from $N_{e_{2}}$ 's point of view, since the driving force is to have followers beyond the use regions of the prefollowers. The solution is to pick a new follower $\hat{x}$ which we must attach to some prefollower $\hat{y} \neq y(x)$ (since we must still respect the $e_{1}$ commitment). Since we wish now to respect $e_{1}$ and $e_{2}$ commitments we would like $\hat{x}$ to exceed both $u\left(\left[e_{1}\right](A \oplus \sigma ; \hat{y})\right)$ and $u\left(\left[e_{2}\right](A \oplus \sigma ; \hat{y})\right)$. To do this we need to have already seen $l\left(e_{1}, t\right)>\hat{y}$ some $t \leq \hat{s}$. Our basic idea is to set aside as many potential prefollowers as we will need in advance and only act when $l(e, s)$ exceeds all of them. Then if we ever need to switch we will know that previous $N_{e}$ commitments remain respected.

We now give the formal details of the argument. First to each $P_{e}$ for $e \in \omega$ we assign an entourage of prefollowers

$$
E(e)=\{\langle e, 1\rangle, \ldots,\langle e, e+3\rangle\} .
$$


In the course of the construction numbers in $E(e)$ may be used or unused (or also cancelled). If $x \in E(e)$ and $x$ is used and uncancelled then $x$ is attached to some follower $y$ of $P_{e}$. Initially all of $E(e)$ except $\langle e, 1\rangle$ are unused.

We shall say that $P_{e}$ requires attention at stage $s+1$ if $A_{s} \cap W_{e, s}=\varnothing$ and $x \in W_{e, s}$ where $x$ is the current follower of $P_{e}$.

Construction.

Stage 0. Declare each $P_{j}$ for $j \in \omega$ as not $e$-confirmed for all $e \leq j$. Assign $\langle j, 0\rangle$ as a follower of $P_{j}$ targeted for $A$ and attached to $\langle j, 1\rangle$. Declare $\langle j, 1\rangle$ as used.

Stage $s+1$.

Step 1. For each unsatisfied $P_{j}$ and each $e \leq j$ if $j \leq s$ and if

(i) $l(e, s)>\langle j, j+3\rangle$, and

(ii) $P_{j}$ is not yet $e$-confirmed,

declare $P_{j}$ as $e$-confirmed. Find the least unused member $\langle j, i\rangle$ of $E(j)$. (This will exist.) Declare $\langle j, i\rangle$ as used. Appoint $x=\langle j, s+1\rangle$ as a follower of $P_{j}$. Declare $y(x)=\langle j, i\rangle$ as attached to $x$. Cancel the previous follower of $P_{j}$ together with its prefollower.

Step 2. For each $j \leq s$ if $P_{j}$ requires attention find the appropriate follower $x$ and enumerate $x$ into $A_{s+1}-A_{s}$ and $y(x)$ (x's prefollower) into $D_{s+1}-D_{s}$.

End of Construction.

(2.4) LEMMA. All the $P_{e}$ are met and not all of the members of $E(e)$ are used.

PROOF. It is clear that $P_{e}$ always has a follower provided we don't run out of prefollowers. We need to reset $P_{e}$ 's follower at most once for each $N_{j}$ with $j \leq e$, and so most $e+1$ times. Thus at most $e+2$ members of $E(e)$ are used. Once we reach a stage after which step 1 never again pertains to $P_{e}, P_{e}$ will have a final follower $x$. For this follower, as usual, either $P_{e}$ never receives attention (and so $\bar{W}_{e} \neq A$ by fiat) or step 2 pertains to $x$ ensuring $W_{e, s} \cap A_{s} \neq \varnothing$.

(2.5) LEMMA. All the $N_{e}$ are met.

Proof. Let $B$ be any set and suppose $[e](A \oplus B)=D$. Then $l(e, s) \rightarrow \infty$ since the appropriate initial segments $\sigma$ of $B$ will satisfy the definition for $l(e, s)$. We must show that $A \leq_{\mathrm{tt}} B$. Let $s_{0}$ be a stage such that

$$
\forall s \geq s_{0} \forall j<e\left(P_{j} \text { does not receive attention at stage } s\right) \text {. }
$$

Our procedure is inductive. Let $z$ be given. Suppose $A[z-1] \leq_{\mathrm{tt}} B$. Now numbers may enter $A$ after stage $s_{0}$ only if they follow some $P_{j}$ for some $j \geq e$. Let $s_{1}=\max \left\{z, s_{0}\right\}$. If $z$ does not follow some $P_{j}$ for $j \geq e$ at stage $s_{1}$ then $z \in A$ iff $z \in A_{s_{1}}$. Assuming $z$ follows $P_{j}$, say, find the least stage $s_{2}>s_{1}$ such that one of the following options holds.

(i) $P_{j}$ is $e$-confirmed at stage $s_{2}$,

(ii) $z \in A_{s_{2}}$,

(iii) $W_{e, s} \cap A_{s} \neq \varnothing$, or

(iv) $z$ is cancelled.

If (ii), (iii) or (iv) hold then $z \in A$ iff $z \in A_{s_{2}}$. If (i) holds we proceed as follows. Find the stage $t \leq s_{2}$ at which $z$ is appointed. By (i) above as $z$ is uncancelled $P_{j}$ is $e$-confirmed at stage $t$. This means $z$ is given a prefollower $y(z)=\langle j, i\rangle$ for some $i$ with $l(e, t)>\langle j, i\rangle$ and

$$
z>u([e](A \oplus B ;\langle j, i\rangle))
$$


Now $z \in A$ iff $\langle j, i\rangle \in D$ and by (2.7) (if case (i) holds) we see

$$
z \in A \quad \text { iff }[e](A[z-1] \oplus B ;\langle j, i\rangle)=1 .
$$

Hence by induction $B$ can tt-compute $A[z]$ and hence $A \leq_{\mathrm{tt}} B$.

(2.6) LeMma. $A \leq_{m} D$.

PROOF. To compute if $z \in A$, see if $z$ is a follower by stage $z$. If not then $z \notin A$. If $z$ is a follower then $z$ has a prefollower $y(z)$. Then $z \in A$ iff $y(z) \in D$.

There is nothing special here about tt-reductions. We remark that the same proof also shows:

(2.7) COROLLARY [1]. There exist r.e. wtt-degrees with the global anticupping property.

We remark that by using an infinite collection of $\{E(e)\}$ for each $P_{e}$ in place of $E(e)$ a standard permitting argument (on $D$ ) shows.

(2.8) COROLLARY [1]. Each nonzero r.e. wtt-degree has the global anticupping property.

PROOF. Left to reader.

Of course (2.8) fails in the r.e. tt-degrees since Kobzev [7] has constructed a minimal r.e. tt-degree. Jeanleah Mohrherr [8] has asked the related question of the extent which Friedberg's [3] completeness criterion holds in $\mathscr{D}_{\mathrm{tt}}$. In [8], Mohrherr showed (for $\mathscr{D}_{\mathrm{tt}}$ )

$$
\forall \mathbf{a} \geq \mathbf{0}^{\prime} \exists \mathbf{b}\left(\mathbf{b}^{\prime}=\mathbf{a}\right)
$$

The question is whether there exists a tt-degree $\mathbf{d}\left(=\mathbf{0}^{\prime}\right.$ ?) such that

$$
\forall \mathbf{a} \geq \mathbf{d} \exists \mathbf{b}(\mathbf{b}<\mathbf{a} \& \mathbf{b} \cup \mathbf{d}=\mathbf{a})
$$

In view of our results, I conjecture that (2.10) fails for $\mathscr{D}_{t t}$.

3. $M$-tops. Our result for this section is to solve Odifreddi's question [9, Problem 11].

(3.1) THEOREM. There exists an r.e. tt-degree without greatest r.e. $m$-degree.

ProOF. We shall build $A=\bigcup_{s} A_{s}$ with auxiliary r.e. sets $B_{e}=\bigcup_{s} B_{e, s}$ to satisfy the requirements (taken over 3 -tuples $\left\langle[e], W_{e}, \gamma_{i}\right\rangle$ )

$$
R_{e, i}:[e](A)=W_{e} \text { implies } \quad B_{e} \leq_{\mathrm{tt}} A \text { and } \rightarrow\left(B_{e} \leq_{m} W_{e} \text { via } \gamma_{i}\right) .
$$

The reader should note that meeting all the $R_{e, i}$ gives (3.1). For suppose $W_{e}$ is an r.e. set of greatest r.e. $m$-degree in tt-degree of $A$. Now as $B_{e} \leq_{\mathrm{tt}} A, B_{e} \oplus A \equiv_{\mathrm{tt}} A$. Since $B_{e} \Varangle_{m} W_{e}$ in particular $B_{e} \oplus A \Varangle_{m} W_{e}$. In fact, we ensure that $B_{e} \leq_{b t t} A$ with norm 2 . The reduction is

$$
x \in B_{e} \quad \text { iff }\left\{\begin{array}{l}
x \text { is a follower target for } B_{e} \text { be stage } x \text { and } \\
2 x \in A \text { and } 2 x+1 \notin A .
\end{array}\right.
$$

This reduction is predicated, of course, on the assumption that $[e](A)=W_{e}$. Followers of $R_{e, i}$ may be active or passive. If a follower $x$ of $R_{e, j}$ is active (and so targeted for $B_{e}$ ) then if $x$ is cancelled at stage $s$, we automatically enumerate $x$ into $B_{e}$. "Activity" therefore involves a "pending commitment" to $B_{e}$. 
We shall say that $R_{e, i}$ requires attention at stage $s+1$ if $R_{e, i}$ is not (declared) satisfied and one of the following options holds.

(3.2) $R_{e, i}$ has no follower.

(3.3) $R_{e, i}$ has a follower $x$ and

(i) $\gamma_{i, s}(x) \downarrow$

(ii) $l(e, s)>x, \gamma_{i}(x)$; where

$$
l(e, s)=\max \left\{x: \forall y<x\left([e]\left(A_{s} ; y\right)=W_{e, s}(y)\right)\right\}
$$

Construction.

Stage $s+1$. Find a least $\langle e, i\rangle$ such that $R_{e, i}$ requires attention. First cancel the satisfaction of all $R_{j, k}$ for $\langle e, i\rangle\left\langle\langle j, k\rangle\right.$. If $R_{j, k}$ is currently active and $x$ is its follower enumerate $x$ into $B_{j, s+1}-B_{j, s}$. Now cancel all followers of $R_{j, k}$.

Now attack the $R_{e, i}$ by adopting the appropriate case below.

Case 1. (3.2) holds; Assign $x=s$ as a follower of $R_{e, i}$. Declare $R_{e, i}$ as passive.

Case 2. (3.3) holds and $R_{e, i}$ is passive. Define $C_{s}=A_{s} \cup\{2 x\}$.

Subcase (a). $[e]\left(C_{s} ; \gamma_{i}(x)\right)=0$. Set $A_{s+1}=A_{s} \cup\{2 x\}$ and $B_{e, s+1}=B_{e, s} \cup\{x\}$. Declare $R_{e, i}$ as satisfied.

Subcase (b). $\quad[e]\left(C_{s} ; \gamma_{i}(x)\right)=1$. Set $A_{s+1}=A_{s} \cup\{2 x\}$ and $B_{e, s+1}=B_{e, s}$. Declare $R_{e, i}$ as active.

Case 3. (3.3) holds and $R_{e, i}$ is active. Set $A_{s+1}=A_{s} \cup\{2 x+1\}$ and $B_{e, s+1}=$ $B_{e, s}$. Declare $R_{e, i}$ as satisfied.

End of construction.

Verification. The argument is finite injury. Let $s_{0}$ be a stage such that

$$
\forall s>s_{0} \forall m>\langle e, i\rangle\left(R_{m} \text { does not receive attention at stage } s\right) .
$$

By our cancellation procedure we may suppose that $R_{e, i}$ has no follower at stage $s_{0}$. Now if $R_{e, i}$ is to fail then $l(e, s) \rightarrow \infty$.

At some stage $s_{1}>s_{0}, R_{e, i}$ receives attention and gets a follower $x$. If $R_{e, i}$ is to fail, then (3.3) must hold at some stage $s_{2}>s_{1}$. At stage $s_{2}$ we see

$$
[e]\left(A_{s_{2}} ; \gamma_{i}(x)\right)=W_{e, s}\left(\gamma_{i}(x)\right) .
$$

By our cancellation procedure, the only numbers (possibly) $<s$ which can ever enter $A-A_{s_{2}}$ are $2 x$ and $2 x+1$. Hence the only numbers (possibly) $<u\left([e]\left(A ; \gamma_{i}(x)\right)\right)$ which can ever enter $A-A_{s_{2}}$ are $2 x$ and $2 x+1$. Now if subcase (a) holds then

$$
[e]\left(A ; \gamma_{i}(x)\right)=0=W_{e}\left(\gamma_{i}(x)\right)
$$

and $\neg\left(B_{e} \leq_{m} W_{e}\right.$ via $\left.\gamma_{i}(x)\right)$ since $B_{e}(x)=1$ and $\gamma_{i}(x) \notin W_{e}$.

If subcase (b) holds we set $A_{s+1}=A_{s} \cup\{2 x\}$ and activate $x$. Since $[e]\left(C_{s} ; \gamma_{i}(x)\right)$ $=1$, when (3.3) next (say at stage $s_{2}>s_{2}$ ) pertains to $R_{e, i}$ we must have

$$
[e]\left(A ; \gamma_{i}(x)\right)=1=W_{e, s_{3}}\left(\gamma_{i}(x)\right) .
$$

But now $\gamma_{i}(x) \in W_{e, s_{3}}$ and so $\gamma_{i}(x) \in W_{e}$ as $W_{e}$ is r.e. But now we win since $x \notin B_{e}$ and $\gamma_{i}(x) \in W_{e}$. It is clear that $B_{e} \leq_{t t} A$ if $l(e, s) \rightarrow \infty$ and so all the $R_{e, i}$ are met.

(3.5) Variations and comments.

The reader should note that we win above for one of two reasons. In subcase (a) by enumerating $2 x$ into $A$ we cause $[e](A)$ to believe $\gamma_{1}(x) \notin W_{e}$ yet enumerating $x$ into $B_{e}$ causes $\gamma_{i}(x) \in W_{e}$. In subcase (b) we first set $A$ so that $[e](A)$ believes 
$\gamma_{i}(x) \in W_{e}$ but does not enumerate $x$ into $B_{e}$. Thus once we see $\gamma_{i}(x) \in W_{e, s}$ at some $s$ we then use $2 x+1$ to allow us to keep $x$ out of $B_{e}$ while $\gamma_{i}(x) \in W_{e}$.

The "punch line" here is that once $\gamma_{i}(x)$ enters $W_{e}$ it cannot be retracted. Let $n$ be given. An easy modification (using a larger norm for the $B_{e} \leq_{\mathrm{btt}} W_{e}$ ) will construct an r.e. tt-degree with no $n$-r.e. $m$-degree exceeding all r.e. $m$-degrees. By dovetailing and using full tt-reductions, it is also possible to construct an r.e. tt-degree a with no $k$-r.e. $m$-degree exceeding all r.e. $m$-degrees in a for any $k$. I do not know if all nonzero r.e. $T$-degrees contain r.e. tt-degrees without r.e. $m$ tops. We should point out that Rogers and Jockusch (cf. [12]) have shown that all tt-degrees contain a greatest $m$-degree. Thus the restriction "r.e. $m$-degree" is necessary. Finally it seems conceivable that the methods of this section might be useful in solving the following question implicit in [2]: Is there tt-topped r.e. $T$-degree that is not $m$-topped?

ADDED IN PROOF. The result above can be extended to construct an r.e. $T$ degree $\mathbf{a}$ such that if $\mathbf{b}$ is any r.e. tt-degree within $\mathbf{a}$, then $\mathbf{b}$ has no greatest r.e. $m$-degree. See [13].

\section{REFERENCES}

1. R. G. Downey, $\Delta_{2}^{0}$ degrees and transfer theorems, Illinois J. Math. 31 (1987), 419-427.

2. R. G. Downey and C. G. Jockusch, T-degrees, jump classes, and strong reducibilities, Trans. Amer. Math. Soc. 301 (1987), 103-136.

3. R. Friedberg, A criterion for completeness of degrees of unsolvability, J. Symbolic Logic 22 (1958), 159-160.

4. P. Fejer and R. Shore, Embeddings and extensions of embeddings in the r.e. $t$ t and wtt-degrees, Recursion Theory Week (Proceedings, Oberwolfach 1984) (Eds., H. Ebbinghaus, G. Müller and G. Sacks), Lecture Notes in Math., vol. 1141, Springer-Verlag, 1985, pp. 121-140.

5. L. Harrington, Plus cupping in the recursively enumerable degrees, handwritten notes, 1978.

6. C. G. Jockusch, Relationships between reducibilities, Trans. Amer. Math. Soc. 142 (1969), 229-237.

7. G. N. Kobzev, On tt-degrees of r.e. T-degrees, Mat. Sb. 106 (1978), 507-514.

8. J. Mohrherr, Density of a final segment of the truth table degrees, Pacific J. Math. 115 (1984), 409-419.

9. P. Odifreddi, Strong reducibilities, Bull. Amer. Math. Soc. (N.S.) 4 (1981), 37-85.

10. Classical recursion theory, (to appear).

11. D. Posner, The upper semilattice of degrees $\leq \mathbf{0}^{\prime}$ is complemented, J. Symbolic Logic 46 (1981), $705-713$.

12. H. Rogers, Theory of recursive functions and effective computability, McGraw-Hill, New York, 1967.

13. R. G. Downey, Recursively enumerable $m$ - and tt-degrees II: the distribution of singular degrees, Arch. Math. Logik (to appear).

Department of Mathematics, Victoria University at Wellington, Private BaG, WELlington, NEW ZEALAND 